\title{
Miocardiopatia de Takotsubo: Características e Mecanismos Etiológicos, uma revisão narrativa-crítica
}

\author{
Takotsubo's Cardiomyopathy: Characteristics and Etiologic Mechanisms, a critical- \\ narrative review \\ Miocardiopatía de Takotsubo: Características y Mecanismos Etiológicos, una revisión \\ narrativa-crítica
}

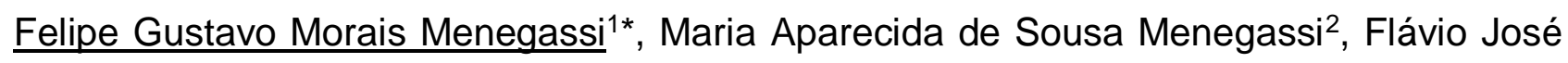
Dutra de Moura'.

\begin{abstract}
RESUMO
Objetivo: Demonstrar, por meio de uma revisão narrativa-crítica, as principais evidências sobre as condições fisiopatológicas da Miocardiopatia de Takotsubo, com base nos mecanismos etiológicos e nas teorias atuais propostas. Métodos: Revisão de literatura a partir de artigos científicos das bases PubMed, LILACS e Scielo. Foram utilizados descritores específicos para obtenção de trabalhos publicados entre os anos 2013 e 2018. Foram incluídos os estudos com base nas características clínicas e excluídos os estudos sem publicações completas e sem estudo em seres humanos. Resultados: Com a adoção dos critérios de inclusão, 238 artigos foram identificados. Destes, 68 artigos se destacaram após uso do critério de exclusão. Do total de artigos selecionados, 27 compõem este estudo por razão de análise minuciosa dos seus conteúdos. Os trabalhos analisados têm seus estudos conduzidos em diversas regiões do mundo, com destaque nos Estados Unidos e Europa. Conclusão: A Miocardiopatia de Takotsubo possui características já bem definidas pela literatura científica, o que permite estabelecer seu diagnóstico. Contudo, percebe-se, ainda, muita incerteza frente aos mecanismos etiológicos e terapêutica definitiva desta identidade diagnóstica.
\end{abstract}

Palavras-chave: Cardiomiopatia, Choque Cardiogênico, Infarto do Miocárdio, Disfunção Ventricular.

\begin{abstract}
Objective: To demonstrate, through a narrative-critical review, the main evidences about the pathophysiological conditions of Takotsubo's Cardiomyopathy based on the etiological mechanisms and current theories proposed. Methods: Literature review from the scientific articles PubMed, LILACS and Scielo. Specific descriptors were used to obtain works published between the years 2013 and 2018. The studies were included based on the clinical characteristics and excluded based on the absence of complete publications and on the absence of study in humans. Results: With the adoption of inclusion criteria, 238 articles were identified. Of these, 68 articles were highlighted after using the exclusion criteria. Of the total of articles selected, 27 compose this study, due to the detailed analysis of its contents. The studies analyzed have been conducted in several regions of the world, with emphasis in the United States and Europe. Conclusion: Takotsubo's cardiomyopathy has characteristics already well defined by the scientific literature, which allows to establish its diagnosis. However, there is still a great deal of uncertainty regarding the etiological and definitive therapeutic mechanisms for this diagnostic identity.
\end{abstract}

Key words: Cardiomyopathy, Shock, Cardiogenic, Myocardial Infarction, Ventricular Dysfunction.

1 Centro Universitário do Planalto Central (Uniceplac), Gama - DF. *E-mail: moraisfelipe029@gmail.com. 2 Universidade Católica de Brasília, Taguatinga - DF. 


\section{RESUMEN}

Objetivo: Demostrar, por medio de una revisión narrativa-crítica las principales evidencias sobre las condiciones fisiopatológicas de la Miocardiopatía de Takotsubo con base en los mecanismos etiológicos y en las teorías actuales propuestas. Métodos: Revisión de literatura a partir de artículos científicos de las bases PubMed, LILACS y Scielo. Se utilizaron descriptores específicos para la obtención de trabajos publicados entre los años 2013 y 2018. Se incluyeron estudios basados en las características clínicas y excluidos los estudios sin publicaciones completas y sin estudio en seres humanos. Resultados: Con la adopción de los criterios de inclusión 238 artículos fueron identificados. De estos, 68 artículos se destacaron después del uso del criterio de exclusión. Del total de artículos seleccionados, 27 componen este estudio, por razón de análisis minucioso de sus contenidos. Los trabajos analizados tienen sus estudios conducidos en diversas regiones del mundo, con destaque en los Estados Unidos y Europa. Conclusión: La Miocardiopatía de Takotsubo posee características ya bien definidas por la literatura científica, lo que permite establecer su diagnóstico. Sin embargo, se percibe aún mucha incertidumbre frente a los mecanismos etiológicos y terapéutica definitiva de esta identidad diagnóstica.

Palabras clave: Cardiomiopatía, Choque Cardiogénico, Infarto del Miocardio, Disfunción Ventricular.

\section{INTRODUÇÃO}

A Miocardiopatia de Takotsubo (MT) foi primeiramente analisada em 1990 pelo Dr. Hikaru Sato, que percebeu a síndrome como uma patologia gerada por gatilhos prolongados de estresses físicos e/ou emocionais. A denominação "Takotsubo" dada pelo cientista tem como referência a morfologia do coração dos portadores desta enfermidade. Ao observar os resultados da ventriculografia esquerda destes pacientes, percebe-se que o coração é análogo a um "tsubo", que em japonês remete a um jarro característico de abertura estreita e corpo amplo. Esses jarros eram muito utilizados por pescadores na pesca de (BARROS et al., 2017; MACIEL et al., 2013).

A MT, também denominada de Síndrome do Coração Partido (SCP), geralmente surge como diagnóstico diferencial de outras entidades patológicas, tais como: infarto agudo do miocárdio (IAM), miocardite e/ou feocromocitoma. Somente após pensa-se em MT como um diagnóstico de exclusão. Essa enfermidade já foi referenciada como miocardiopatia de stress (MS), discinesia apical transitória cardíaca do ventrículo esquerdo (VE) e até de síndrome do balonamento apical (SBA) (FEVEREIRO et al., 2015; MENEZES et al., 2016).

A MT é decorrente de fatores estressores físicos ou emocionais que, a longo prazo, levam a distúrbios eletrocardiográficos próximos aos encontrados na síndrome coronariana aguda (SCA), acarretando aumento dos marcadores enzimáticos relacionados à lesão miocárdica, mas que tendem a reduzir até os níveis fisiológicos dentro de alguns dias, o que não ocorreria na SCA (BARROS et al., 2017).

Na literatura, há uma dificuldade em delinear os aspectos fisiopatológicos da MT. Todavia, já se percebe a sua forte relação com a disfunção miocárdica devido aos prejuízos na microcirculação cardíaca que levam à isquemia dos tecidos cardíacos ou aos danos causados pelos elevados níveis de catecolaminas geradores de efeitos cardiotóxicos (ELIKOWSKI et al., 2017; ONO e FALCAO, 2016; LEE et al., 2016; WANG et al., 2015).

Segundo Fevereiro et al. (2015), a MT possui prevalência indefinida. Contudo, estima-se uma associação de $1-2 \%$ dessa patologia com a SCA. Vale salientar que 4 em 5 pacientes são mulheres, preponderantemente a partir dos 60 anos de idade.

A etimologia da doença está relacionada a 'situações-gatilho', tais como: participação de eventos estressores, presenciar familiares em procedimentos cirúrgicos, estresse pós-traumático, toxicidade a drogas, morte de familiares, catástrofes naturais, assédio moral, desmame de medicamentos, ansiedade pré-cirúrgica etc. (YERASI et al., 2016; LIMON et at., 2016; BROOKS et al., 2018).

Diante de tantos novos artigos produzidos acerca da MT, este estudo vem demonstrar, por meio de uma revisão narrativa-crítica, as principais evidências sobre as suas condições fisiopatológicas com base nos mecanismos etiológicos e teorias atuais propostas.

REAS/EJCH | Vol. Sup. 22 | e380 | DOI: https://doi.org/10.25248/reas.e380.2019 Página 2 de 7 


\section{MÉTODOS}

O estudo desenvolvido nesta revisão de literatura foi fundamentado com os resultados obtidos nas pesquisas bibliográficas realizadas nas bases de dados PubMed, LILACS e SciELO. Para seleção dos artigos, foram utilizados os descritores: Cardiomiopatia de Takotsubo (Takotsubo Cardiomyopathy), Choque Cardiogênico (Shock, Cardiogenic), Infarto do Miocárdio (Myocardial Infarction) e Disfunção Ventricular (Ventricular Dysfunction). Por meio do PubMed, LILACS e SciELO foram obtidos 127, 107 e 4 artigos, respectivamente. Todos conforme o seguinte planejamento de busca: Cardiomiopatia de Takotsubo AND infarto do miocárdio NOT infarto do miocárdio em jovens (Takotsubo Cardiomyopathy AND myocardial infarction NOT myocardial infarction in young adults). Foram utilizados os critérios de seleção: publicação entre os anos 2013 e 2018, disponibilidade de texto completo para estudo e aderência à temática "cardiomiopatia de takotsubo" em pelo menos uma de suas características clínicas. Dessa forma, foram excluídos 79, 76 e 3 artigos, respectivamente. Foram colhidos para análise 48, 31 e 1 artigos, respectivamente. Destes, 11 artigos do PubMed e 1 do LILACS foram excluídos por não realizarem estudos em seres humanos. Foram selecionados para análise posterior 37 artigos do PubMed, 30 artigos do LILACS e 1 artigo do SciELO. Devido à heterogeneidade dos conteúdos, desenvolveu-se estudo exploratório/descritivo com posterior revisão narrativa-crítica dos achados pertinentes.

\section{RESULTADOS E DISCUSSÃO}

Os achados bibliográficos geraram 68 artigos. A partir desses resultados, foram efetuados estudos exploratório/descritivos de todos os resumos e conteúdo dos trabalhos selecionados, buscando encontrar aqueles mais pertinentes para desenvolvimento da presente pesquisa. Após revisão dos achados, foram destacados 27 artigos aptos para subsidiarem a elaboração desta revisão de literatura.

\section{Etiologia e Patogênese}

Na literatura, encontram-se apenas algumas características específicas da MT. Ainda há incertezas com relação à etiopatogenia da doença. Devido a esse fator importante, existem diversas alternativas quanto à abordagem especializada da enfermidade em regime ambulatorial (LIMON et al., 2016; WANG et al., 2015).

Segundo Barros et al. (2017), a origem da patologia decorre do início abrupto de sintomas associados a distúrbios eletrocardiográficos semelhantes aos encontrados na SCA. O quadro clínico apresenta associação da lesão miocárdica com aumento de enzimas específicas do órgão, sendo que ao longo de muitos dias o quadro clínico tem reversão completa, o que não ocorreria na SCA.

Fevereiro et al. (2015) sugerem a ocorrência de MT após fatores estressores físicos ou emocionais, bem como surtos de enfermidades agudas ou em usuários de drogas ilícitas. Os autores também correlacionam a SCP à SCA, sendo que aproximadamente 1-2\% desses pacientes são diagnosticados com MT.

Muitas alterações são observadas nos portadores da MT. Entretanto, alguns autores sugerem que os principais distúrbios fisiopatológicos da doença são lesão do miocárdio e necrose dos tecidos cardíacos decorrentes de elevados níveis de catecolaminas (RODRIGUES et al., 2015; ELIKOWSKI et al., 2017).

A fisiopatologia da MT também permanece incerta. Contudo, existem relatos de prováveis fatores-gatilho. Dentre eles, é possível perceber: hiperfunção adrenoreceptora, elevada ativação dos receptores $\beta$, sinalização epinefrínica, distúrbios microvasculares, ações tóxicas no miocárdio decorrentes de elevados índices de catecolaminas, espasmos de vasos de grande calibre cardíaco e placas geradoras de obstrução (MENEZES et al., 2016; ONO e FALCÃO, 2016; WANG et al., 2015).

Ainda é pauta de discussão o rompimento das placas ateroscleróticas e o desenvolvimento da MT. Existem relatos dessas placas nas artérias coronárias de muitos enfermos, mas também ocorre uma alta prevalência nesta faixa etária com aterosclerose, podendo acarretar confusão quanto a influência destas na etiologia da doença, pois tanto a MT quanto o acometimento das placas são comuns nesta fase da vida (AMARAL et al., 2014; EITEL et al., 2016; MACIEL et al., 2013).

REAS/EJCH | Vol. Sup. 22 | e380 | DOI: https://doi.org/10.25248/reas.e380.2019 Página 3 de 7 
Nos estudos de Eitel et al. (2016), aproximadamente $25 \%$ dos portadores da MT apresentaram algum tipo de fibroatenoma de placa fina fragilizado e predisposto a rompimento com geração de trombose. Devido a esse fator, a longo prazo, referem-se elevadas taxas de mortalidade. Diante desses achados, a literatura recomenda o acompanhamento dos níveis de colesterol de cada paciente com foco na sua redução, pois refletem baixos níveis de placas ateroscleróticas e são um fator protetivo na MT, inclusive naqueles sem comorbidades cardiovasculares.

\section{Apresentação clínica}

A SCP tem apresentação clínica muito próxima da SCA na sua fase aguda. Por possuírem muitos sintomas semelhantes, é comum a incerteza no seu diagnóstico. Os sinais e sintomas da MT são: dor torácica (comum na região retroesternal), dispneia, arritmias, palpitação e síncope; hipertensão, choque cardiogênico e convulsões atingem 5-10\% dos pacientes; dor abdominal e vômitos acometem 2-3\% dos casos. Como manifestações clínicas inespecíficas, são descritas fraquezas, náuseas, vômitos, febre e diaforese. Quanto ao gênero, as mulheres se queixam mais de dor retroesternal. Em ambos os sexos são queixas igualmente reportadas à dispneia e à síncope (SCHNEIDER e SECHTEM, 2016; JUNG et al., 2016; WANG et al., 2015). Contudo, mulheres tendem a ser mais acometidas pela doença após surtos de exaustão física ou emocional. Esses gatilhos estressores tendem a elevar os níveis de catecolamina gerando cardiotoxicidade. Também são associados a alterações metabólicas, distúrbios de capilares cardíacos e espasmo de vasos coronarianos (RODRIGUES et al., 2015; ONO e FALCÃO, 2016).

\section{Diagnóstico}

Uma das tentativas de se estabelecer um diagnóstico definitivo é por meio dos critérios modificados e propostos pela Clínica de Mayo. Esses critérios são muito referendados na literatura científica, o que reforça a sua relevância no desfecho diagnóstico (SILVA et al., 2015; RODRIGUEZ et al., 2018).

Quatro critérios de diagnóstico necessários (Clínica de Mayo): 1. Hipocinesia, discinesia ou acinesia transitória dos segmentos médios e apicais do VE, com ou sem envolvimento apical; com alterações da contratilidade da área de vascularização correspondente a mais do que uma artéria coronária; um gatilho estressante é muitas vezes presente. 2. Ausência de doença coronária obstrutiva ou evidência angiográfica de ruptura aguda da placa. 3. Alterações eletrocardiográficas de novo (supradesnivelamento do segmento ST e/ou inversão da onda T) ou elevação modesta da troponina cardíaca. 4. Ausência de feocromocitoma ou miocardite (BARROS et al., 2017; ONO e FALCÃO, 2016; CHAZAL et al. 2018).

A lesão cardíaca ou sua disfunção podem ter etiologia devido a: hemorragia subaracnoidea; outros tipos de hemorragia cerebral; traumatismo cranioencefálico agudo ou acidente vascular encefálico (AVE) não hemorrágico. A lesão nos vasos coronários é, muitas vezes, associada à cardiomiopatia por estresse neurogênico (CEN), SCP, miocárdio atordoado neurogênico ou simplesmente lesão neurocárdica. Ainda há muita incerteza na definição da lesão miocárdica e seus desdobramentos (LEE et al., 2016; RANIERI et al., 2018).

A CEN, por exemplo, possui causa, evolução e desenvolvimento semelhante à MT. Ambas as patologias possuem analogia quanto às lesões cardíacas por excesso de catecolaminas e necrose da banda de contratilidade do coração. Todavia, a CEN é considerada uma alteração miocárdica com característica da MT associada a uma patologia cerebrovascular. O quadro clínico e os critérios diagnósticos da CEN permanecem ainda incertos (LEE et al., 2016).

Segundo Ali et al. (2018), a MT, a SCA e o miocárdio atordoado neurogênico (MAN) possuem muitas particularidades em comum. Dentre essas características, é possível citar: lesões cardíacas, distúrbios no VE, aumento da troponina e distúrbios eletrocardiográficos. Dessa forma, somente o quadro clínico muitas vezes não é suficiente para desfecho diagnóstico. O MAN ocorre quando há distúrbios cardíacos decorrentes de acometimentos neurológicos como um AVE, por exemplo. Determinados desarranjos no sistema nervoso refletem nos tecidos cardíacos gerando lesões e necroses. Para diferenciar MAN de outras identidades diagnósticas são necessários exames complementares.

REAS/EJCH | Vol. Sup. 22 | e380 | DOI: https://doi.org/10.25248/reas.e380.2019 Página 4 de 7 


\section{Diagnóstico Diferencial}

A literatura científica vigente salienta que a MT não é fácil de diagnosticar. Muitas patologias têm quadro clínico semelhante, o que confunde e gera incertezas durante o desfecho diagnóstico. Entretanto, existem pontos importantes que diferenciam cada patologia e ajudam a sanar muitas incertezas (YALTA et al., 2018).

Miocardite. Ocorre muitas vezes com dor torácica associada a quadro clínico de insuficiência cardíaca. A ressonância magnética cardíaca (RMC) é um exame muito relevante para ratificação da doença. Interessante ressaltar que a literatura sugere o realce tardio do gadolínio (RTG) na RMC. Este é bastante favorável para diferenciar miocardite da SCP. O gadolínio é ordinariamente não encontrado nos portadores de MT. Porém, na miocardite, epicárdio e miocárdio são comumente destacados pelo RTG. Nessa patologia é comum o aumento dos biomarcadores cardíacos e disfunções no eletroencefalograma. Contudo, quando se percebe balonismo apical ou, em outras circunstâncias, hipocinesia basal, o quadro de disfunção da miocardite se torna um diagnóstico impensável (CHAZAL et al., 2018).

Feocromocitoma. Pode ser causa de disfunção do ventrículo cardíaco por meio do aumento das catecolaminas, devido a uma tumoração neuroendócrina que passa a produzir catecolaminas excessivamente. Essa tumoração tem etimologia nas células cromafins da medula adrenal. Os distúrbios de contratilidade segmentar sem presença de danos ao miocárdio estão comumente relacionados à miocardite, feocromocitoma ou outras patologias. No caso do feocromocitoma, ainda há um aumento da pressão arterial, cefaleia, sudorese, dor torácica e arritmias. As catecolaminas são responsáveis por diversos transtornos a nível cardiovascular. O feocromocitoma gera quadro clínico predominantemente crônico. Essa patologia ainda pode coexistir com outras enfermidades, como a SCP, devido ao aumento crônico das catecolaminas (CHAZAL et al., 2018; MACIEL et al., 2013).

Síndrome Coronariana Aguda. As placas ateroscleróticas frequentemente geram desdobramentos agressivos a longo prazo. Além disso, é preciso conhecer bem a MT, pois esta pode vir sobreposta à SCA com relação aos sinais, sintomas e alguns exames cardiológicos. Um exame muito útil diante de diagnósticos etiológicos próximos é a angiografia coronariana que avalia a extensão e localidade do dano às artérias coronárias (CHAZAL et al., 2018).

Dentre os portadores de SCA, a angina vasoespástica (AV) é uma das causas mais relevantes de IAM epicárdico com presença de aterosclerose coronária não obstrutiva. A AV atua com espasmos em artérias coronárias epicárdicas de forma localizada, multifocal ou multivascular. Os espasmos acontecem em qualquer vaso coronário. Todavia, vasos com placas ateroscleróticas aumentam a chance e severidade do quadro. A $\mathrm{AV}$ tem essa denominação devido à dor torácica característica em pacientes em repouso à noite ou na primeira parte da manhã. A MT não segue esse padrão de dor. Além disso, a AV tem quadro clínico arrastado, muitas vezes, por semanas ou meses, e com aumento do segmento ST. Vale salientar que é possível acontecer SCA com MT, ou seja, as duas patologias podem ocorrer em situações de lesões cardíacas específicas, levando a estudo clínico e laboratorial específico (CHAZAL et al., 2018).

\section{Diferenças entre os sexos na Miocardiopatia de Takotsubo}

Segundo Sobue et al. (2017), apenas 30\% dos pacientes estudados eram do sexo masculino. Contudo, $80 \%$ dos casos de óbito relacionados à MT eram homens, indicando a alta mortalidade nesse grupo. Na MT, o gênero masculino está adstrito a uma série de eventos adversos responsáveis por um mau prognóstico (distúrbios cardiovasculares, arritmias e falhas cardíacas). Ainda não é possível estabelecer uma razão para o quadro clínico diverso entre os gêneros na MT. Todavia, as comorbidades e severidades a nível cardiovascular têm papel relevante nesse estudo. Além disso, a presença de estrogênio também parece ter influência no desenvolvimento e desdobramento da SCP. Estudos em animais comprovam o fator protetivo do estrogênio contra danos a nível miocárdico. Quando secretado em mulheres depois da menopausa, esse hormônio pode auxiliar em um melhor prognóstico.

A RMC preconiza que os portadores da patologia de Takotsubo, principalmente mulheres mais idosas, têm demasiada exaustão sistólica e diastólica graças a fatores estressores. Justificando, dessa forma, parcialmente a alta prevalência da doença em mulheres e acima de 60 anos (YALTA et al., 2018).

REAS/EJCH | Vol. Sup. 22 | e380 | DOI: https://doi.org/10.25248/reas.e380.2019 Página 5 de 7 
Salmoirago-Blotcher et al. (2016) relataram a existência de características reprodutivas associadas à prevalência da SCP no sexo feminino. Presença de menstruação atípica, quantidade de gestações e uso da terapia de reposição hormonal são situações relacionadas à incidência da MT. Em seu estudo epidemiológico é demonstrado predomínio de acometimentos cardiovasculares somente após a menopausa, o que gera a crer no papel protetivo do estrogênio contra aterosclerose e doenças cardiovasculares.

\section{Características eletrocardiográficas, biomarcadores cardíacos e aspectos imagiológicos}

Rodrigues et al. (2015) trazem a importância de testes laboratoriais para exclusão dos diagnósticos diferenciais e melhor estudo da MT. Segundo os autores, pacientes com MT têm dor na região torácica anterior, secreção excessiva de suor, desconforto respiratório e ECG, indicando possível lesão cardiovascular. A angiografia não apresenta alterações fisiológicas relevantes.

Quanto ao eletrocardiograma, é possível apresentar lesão miocárdica e/ou ter achados inespecíficos. Na fase aguda é comum a presença de elevação do segmento ST, preponderantemente nas derivações de V3 e/ou V6. Contudo, na fase subaguda, a onda T tem grande redução a nível V1 e/ou V6 com alargamento de QT. A ultrassonografia de imagem cardíaca pode apresentar distúrbios da contratilidade do VE (AMARAL et al., 2014; WANG et al., 2018).

Amaral et al. (2014) relataram aumento insidioso dos biomarcadores cardíacos com provável lesão e necrose miocárdica. O IAM frequentemente está relacionado à elevação dos biomarcadores cardíacos, sendo importante para diagnóstico diferencial. Elevação desses marcadores também está relacionada ao estresse físico e a ausência do aumento não é suficiente para descartar a MT. É comum haver elevação da troponina T em 24 horas, com redução gradativa. Outro biomarcador importante é o peptídeo B relacionado a distúrbios do VE.

\section{Tratamento}

Barros et al. (2017) relataram a inexistência de terapêutica padrão para a patologia. Isso decorre do fato de a fisiopatologia ainda não ser bem estabelecida. A terapêutica pode ser dividida em duas fases: na aguda, o objetivo é tratar o quadro clínico que pode ser variável e com graus diferentes de sintomas, e em caso de complicações, as mesmas deverão ser acompanhadas e tratadas; numa segunda fase - caracterizada pela abstenção de complicações -, o quadro clínico é bem mais satisfatório, pois é possível recuperação completa, com normalização ventricular, readequação dos biomarcadores cardíacos, além de regressão completa dos sinais e sintomas. O quadro clínico tende a normalizar em até oito semanas, mas ainda são perceptíveis no ECG traços da doença por anos.

Na MT, os fármacos antitrombóticos e os nitratos precisam ser suspensos, sendo o tratamento de escolha a terapia de suporte. Como as catecolaminas têm papel muito relevante na patologia de Takotsubo, os $\beta$ bloqueadores e os inibidores da enzima de conversão da angiotensina (IECA) são recomendados como terapia específica a nível cardíaco. Não há necessidade do uso de $\beta$-agonistas e vasopressores. Os inotrópicos não são recomendados em decorrência dos relevantes efeitos colaterais. Caso haja choque cardiogênico, o balão intra-aórtico de contrapulsação é uma abordagem a ser considerada, sendo a ventilação mecânica um bom coadjuvante neste processo. Nos casos de hipotensão, o mesmo procedimento será indicado. O profissional deve estar atento à possível obstrução do VE, pois, neste caso, as opções acima devem ser descartadas (AMARAL et al., 2014; RODRIGUEZ et al., 2018; CHAE et al., 2018).

Estudos no âmbito psiquiátrico têm tido pouca relevância na literatura. Contudo, os $\beta$-bloqueadores são muito usados na prevenção e redução de possíveis transtornos mentais em decorrência de forte estresse físico ou emocional. A psicoterapia, dessa forma, tem grande importância como terapia alternativa e como fator protetivo. A anticoagulação pode ser utilizada por um período específico nos casos que apresentem fibrilação atrial ou quando há presença de trombos, e os diuréticos são indicados na insuficiência cardíaca (AMARAL et al., 2014; NAYERI et al., 2018). 


\section{CONSIDERAÇÕES FINAIS}

A MT é uma patologia de acometimento raro com fisiopatologia ainda incerta. Estudos indicam uma forte associação da doença com fatores estressores físicos e emocionais. A etiologia da doença está associada à depressão, transtornos sociais, drogas ilícitas, estresse pós-traumático e algumas doenças agudas. Alguns diagnósticos diferenciais têm destaque com relação a esta identidade diagnóstica, tais como: SCA, miocardite e feocromocitoma. A diferença de gênero desempenha grande papel na incidência, prevalência e prognóstico da MT. Por fim, ao contrário dos seus diagnósticos diferenciais, a Síndrome de Takotsubo não tem tratamento padrão e específico, devendo ser analisado cada caso em particular.

\section{REFERÊNCIAS}

1. ALI A, AHMAD MQ, MALIK MB et al. Neurogenic Stunned Myocardium: A Literature Review. Cureus, 2018; 10(8): e3129.

2. AMARAL WAEF, MIRANDA Z, MIRANDA G et al. Disfunção ventricular apical transitória (Síndrome de Takotsubo): uma revisão de literatura. Arq Catarin Med. 2014; 43(4): 70-76.

3. BARROS J, GOMES D, CARAMELO $S$ et al. Abordagem perioperatória de doente com síndrome de takotsubo. Rev Bras Anestesiol. 2017; 67(3): 321-325.

4. BROOKS JK, WARBURTON G, CLARK BC. Takotsubo Syndrome Following Surgical And Non-Surgical Oral And Maxillofacial Events: Review Of Published Cases. Journal of Oral and Maxillofacial Surgery. 2018.

5. CHAE YK, CHIEC L, ADNEY SK et al. Posterior reversible encephalopathy syndrome and takotsubo cardiomyopathy associated with lenvatinib therapy for thyroid cancer: a case report and review. Oncotarget. 2018; 9:28281-28289.

6. CHAZAL HM, BUONO MG, KEYSER-MARCUS L et al. Stress Cardiomyopathy Diagnosis and Treatment. JACC Vol. 72, No. 16, 2018. 1955-71.

7. ELIKOWSKI W, MALEK-ELIKOWSKA M, KARON J et al. Takotsubo cardiomyopathy after intravenous epinephrine administration following cardiac arrest provoked by pneumoperitoneum - a case report. Pol Med J, 2017; XLII (250); 165-169.

8. EITEL I, STIERMAIER T, GRAF T et al. Optical Coherence Tomography to Evaluate Plaque Burden and Morphology in Patients With Takotsubo Syndrome. J Am Heart Assoc. 2016;5:e 004474.

9. FEVEREIRO MC, SIMÕES MI, LAMPREIA F et al. Miocardiopatia de Takotsubo: na origem do choque cardiogênico. Acta Med Port, 2015; 28(5): 670-673.

10. JUNG JM, KIM JG, KIM JB et al. Takotsubo-Like Myocardial Dysfunction in Ischemic Stroke. A Hospital-Based Registry and Systematic Literature Review. Stroke, 2016; 47:00-00.

11. LEE M, OH JH, LEE KB et al. Clinical and Echocardiographic Characteristics of Acute Cardiac Dysfunction Associated with Acute Brain Hemorrhage. Circ J, 2016; 80: 2026-2032.

12. LIMON JMG, ARANA SI, CARMONA JR et al. Clinical and echocardiographic course in Takotsubo cardiomyopathy: long-term follow-up from a multicenter study. International Journal of Cardiology, 228, 2017; 97-102.

13. MACIEL BA, CIDRAO AAL, SOUSA IBS et al. Pseudoinfarto agudo do miocárdio devido à síndrome da disfunção ventricular apical transitória (síndrome de Takotsubo). Rev Bras Ter Intensiva. 2013; 25(1):63-67.

14. MENEZES MN, SILVA D, ALMEIDA AG et al. Um caso raro de cardiomiopatia de stress (Takotsubo) e enfarte agudo do miocárdio concomitantes. Rev Port Cardiol, 2016; 35:385.

15. NAYERI A, RAFLA-YUAN E, KRISHNAN S et al. Psychiatric Illness in Takotsubo (Stress) Cardiomyopathy: A Review. Psychosomatics, 2018; 01.011.

16. ONO R, FALCAO LM. Takotsubo cardiomyopathy systematic review: Pathophysiologic process, clinical presentation and diagnostic approach to takotsubo cardiomyopathy. International Journal of Cardiology, 2016; 02.012.

17. RANIERI M, FINSTERER J, BEDINI G et al. Takotsubo Syndrome: Clinical Features, Pathogenesis, Treatment, and Relationship with Cerebrovascular Diseases. Current Neurology and Neuroscience Reports, 2018; 18:20.

18. RODRIGUES LBH, BATISTA A, MONTEIRO $F$ et al. Supradesnivelamento do segmento ST durante anestesia geral para cirurgia não cardíaca: um caso de takotsubo. Rev Bras Anestesiol. 2015;65(5):403-406.

19. RODRIGUEZ M, RZECHORZEK W, HERZOG E et al. Misconceptions and Facts about Takotsubo Syndrome. The American Journal of Medicin, 2018; 07.007.

20. Salmoirago-Blotcher E, Dunsiger S, Swales HH et al. Reproductive History of Women With Takotsubo Cardiomyopathy. The American Journal of Cardiology. 2016.

21. SCHNEIDER B, SECHTEM U. Influence of Age and Gender in Takotsubo Syndrome. Heart Failure Clin 12, 2016; 521-530.

22. SILVA MP, VILELA EM, LOPES RL et al. Cardiogenic shock induced by Takotsubocardiomyopathy: A new therapeutic option . Rev Port Cardiol. 2015.

23. SOBUE Y, WATANABE E, ICHIKAWA T et al. Physically triggered Takotsubo cardiomyopathy has a higher in-hospital mortality rate. International Journal of Cardiology, 2017;02.090.

24. YALTA K, YILMAZTEPE M, ZORKUN C. Left Ventricular Dysfunction Takotsubo Cardiomyopathy. Cardiac Failure Review 2018;4(1):14-20.

25. YERASI C, KOIFMAN E, WEISSMAN G et al. Impact of triggering event in outcomes of stress-induced (Takotsubo) cardiomyopathy. European Heart Journal: Acute Cardiovascular Care 1-7, 2016.

26. WANG Q, YU H, JIANG C et al. Cerebral infarction as initial presentation in stress cardiomyopathy. Case report and literature review. Medicine, 2018; 97:20 (e10804).

27. WANG Y, XIA L, SHEN $X$ et al. A New Insight Into Sudden Cardiac Death in Young People. A systematic review of cases of Takotsubo Cardiomyopathy. Medicine, 2015; 94(32):e1174. 